\title{
Modelling and Verifying a Priority Scheduler for an SCJ Runtime Environment
}

\author{
Leo Freitas ${ }^{1(\bowtie)}$, James Baxter $^{2}$, Ana Cavalcanti ${ }^{2}$, and Andy Wellings ${ }^{2}$ \\ 1 Newcastle University, Newcastle upon Tyne, UK \\ leo.freitas@ncl.ac.uk \\ 2 University of York, Heslington, UK \\ jeb531@york.ac.uk
}

\begin{abstract}
Safety-Critical Java (SCJ) is a version of Java suitable for programming real-time safety-critical systems; it is the result of an international standardisation effort to define a subset of the Real-Time Specification for Java (RTSJ). SCJ programs require the use of specialised virtual machines. We present here the result of our verification of the scheduler of the only SCJ virtual machine up to date with the standard and publicly available, the icecap HVM. We describe our approach for analysis of (SCJ) virtual machines, and illustrate it using the icecap HVM scheduler. Our work is based on a state-rich process algebra that combines $\mathrm{Z}$ and CSP, and we take advantage of well established tools.
\end{abstract}

Keywords: Java $\cdot$ SCJ $\cdot$ Circus $\cdot$ Process algebra $\cdot$ FDR

\section{Introduction}

There has been an international effort to make Java and its Runtime Environment (RTE) suitable for safety-critical systems. All proposed extensions to Java have an associated Java Specification Request (JSR), a Reference Implementation (RI) and a Technology Compatibility Kit (TCK). The Safety-Critical Java (SCJ) specification (JSR 302) is an Open Group Standard [20], based on a subset of the Real-Time Specification for Java (RTSJ) [35]. It defines Java services designed for applications requiring certification. It replaces Java's memory model with support for memory regions [34], and its execution model is based on missions and event handlers with a predictable scheduler.

The goal of an RI is to demonstrate the feasibility of implementing a proposed JSR and to illustrate its impact on the standard Java RTE. The RI for JSR 302 consists of the addition of the javax.safetycritical package and a modified JVM. Together these make up the SCJ RTE.

The TCK is a suite of test programs that check that an implementation conforms to a JSR. The SCJ TCK, when available, will provide a degree of confidence in the correctness of an SCJ RTE. It is unlikely, however, that this will be adequate for systems with the highest certification level. For SCJ to become a viable technology, certified runtime environments must become available.

(C) The Author(s) 2016

E. Ábrahám and M. Huisman (Eds.): IFM 2016, LNCS 9681, pp. 63-78, 2016.

DOI: $10.1007 / 978-3-319-33693-0 \_5$ 
The constraints embedded in the SCJ design makes programs amenable to formal analysis. Schedulability analysis techniques [3] can be used to provide evidence that programs meet their deadlines. Ongoing effort to support development, validation, and verification of SCJ programs has already produced results $[6,7,16,24,33]$. The work presented here uses formal methods to increase confidence and provide evidence that an SCJ RTE satisfies its requirements.

SCJ programs cannot run on a standard JVM; they require specialised support for memory regions and preemptive priority-based scheduling. The SCJ RI is under development and will be based on JamaicaVM [18]. To our knowledge, there are currently five SCJVM (virtual machines that support SCJ): Fiji VM [28], icecap HVM (Hardware near Virtual Machine) [32], Ovm (Open Virtual Machine) [1], HVM $\mathrm{TP}_{\mathrm{TP}}$ [21] and PERC Pico [2,29]. Of these, Fiji VM and Ovm are not specific for SCJ, PERC Pico does not conform to the current version of SCJ, and $\mathrm{HVM}_{\mathrm{TP}}$ is based on the icecap HVM.

As far as we know, the only SCJVM that is up to date with the SCJ standard and publicly available is the icecap HVM. Here, we consider the verification of its single-processor scheduler, a core component of an SCJVM. We present a formal model, and establish some of its properties by model checking and theorem proving. This is part of a larger effort to produce a completely verified SCJ RTE. We also present the general approach to construct and analyse formal models of an SCJVM that we use in the verification of the icecap HVM scheduler.

An identification of requirements for an SCJVM and an associated formal model are presented in [4]. The modelling uses Circus [26], a state-rich process algebra that combines $\mathrm{Z}$ and CSP. Circus is a notation for refinement and can be used to compare the requirements in [4] with models of implementation.

In our work, we use Circus processes to specify components of the icecap tools and their integration. Circus has an extension to deal with object-orientation [5]; it is helpful here, since the icecap tools are implemented in Java. We pursue a close match to the implementation structure to provide accurate low-level Circus models. In our approach, the Circus processes define the boundaries of each component of an SCJVM implementation, and their dependencies. Due to the compositionality of refinement, we can analyse these components in isolation. We tackle here a central component of an SCJVM, the scheduler, and identify the assumptions it makes about management of SCJ processes.

In summary, our contributions in this paper are a modelling and analysis technique tailored for an SCJVM, and its application to the scheduler of the only up to date SCJVM publicly available. The discussion of the technique summarises the lessons we have learned in carrying out this case study. In addition, the Circus model itself is of interest for documentation of the icecap tools and for fostering reuse of the icecap scheduler in the implementation of other SCJVMs.

Next, we present background material for our work: SCJ and the icecap tools, and Circus. Section 3 describes our modelling approach. Sections 4 and 5 present our scheduler model and its analysis. We consider related work in Sect.6, and conclude in Sect. 7 considering also future work. 


\section{Preliminaries}

We present SCJ and the icecap tools in Sect. 2.1, and Circus in Sect. 2.2.

\subsection{SCJ and the Icecap Tools}

SCJ places restrictions on the features of Java that can be used, and defines different scheduling and memory models. SCJ has three compliance levels of increasing complexity; the icecap tools support all levels.

An SCJ program is structured as a series of missions, executed sequentially in an order determined by a program-supplied mission sequencer. Each mission manages various schedulable objects: asynchronous event handlers (at levels 0 and 1), and real-time threads and nested mission sequencers (at level 2). A mission execution goes through several phases shown in Fig. 1. First, each of the schedulable objects and any data that may be required for the duration of the mission are initialised. Afterwards, the mission runs until requested to terminate, and then each of the schedulable objects are terminated, any required cleanup is performed, and the mission sequencer runs the next mission.

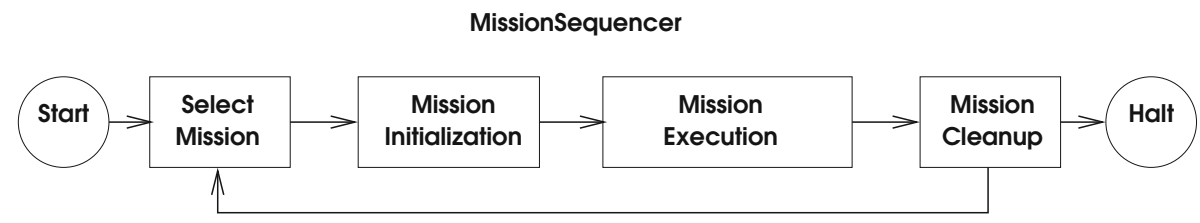

Fig. 1. A diagram showing the phases of mission execution

Asynchronous event handlers can be released periodically at set intervals or aperiodically in response to software requests. Schedulable objects are managed by a priority-based preemptive scheduler. Priority ceiling emulation, whereby a thread has its priority elevated upon taking a lock, prevents problems arising from priority inversion in locking [35]. Support for multiprocessor systems allows schedulable objects to be associated with allocation domains that define the processors in which they are allowed to run.

The icecap tools target embedded systems and precompiles Java bytecode to $\mathrm{C}$, in addition to supplying a lightweight bytecode interpreter. The running of SCJ programs is supported by an implementation of the SCJ API, tightly coupled to the SCJVM. The API implementation and the code that supports it are written in Java, with only the most low-level components written in $\mathrm{C}$ and assembly. In the scheduler, only the task of process switching is written in $\mathrm{C}$ and assembly, with the code to determine which process should run written in Java.

The structure of the scheduler implementation is shown in Fig. 2. The scheduler is triggered by the clock interrupt handler, a singleton instance of ClockInterruptHandler, which implements the interfaces InterruptHandler and 


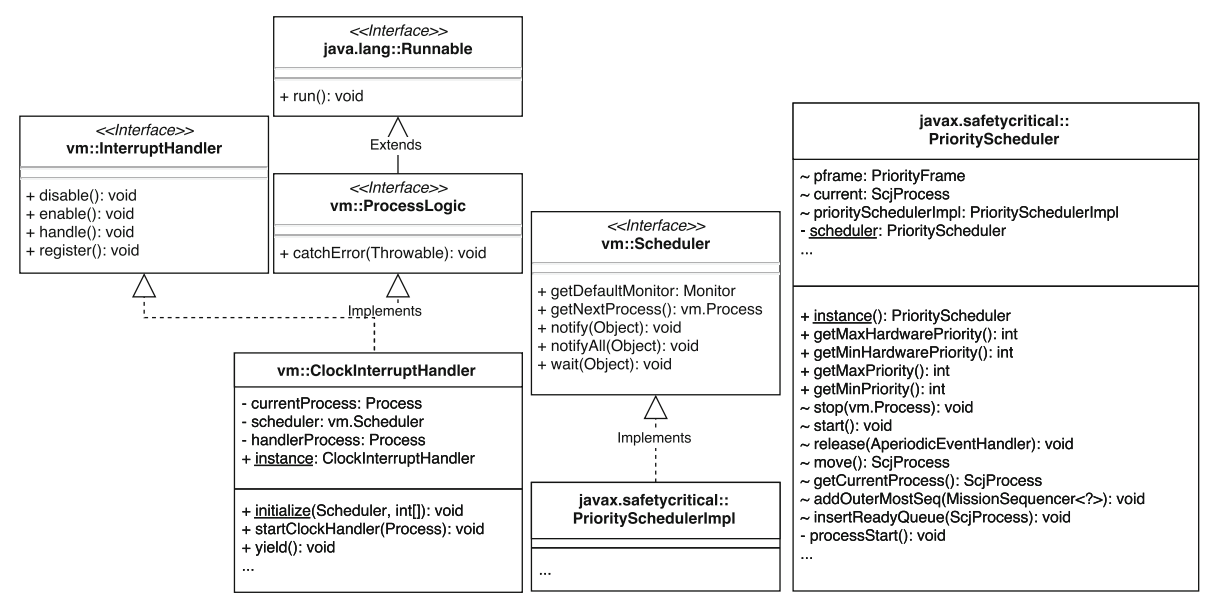

Fig. 2. UML class diagram showing the classes that make up the icecap HVM scheduler

Runnable. A process executing the run() method of the ClockInterruptHandler instance is created when the clock interrupt handler is initialised. Upon receiving a clock interrupt, the icecap HVM calls the clock interrupt handler's handle () method, which switches to the clock interrupt handler's process. The clock interrupt handler then calls the scheduler's getNextProcess() method.

The scheduler itself is an instance of PrioritySchedulerImpl, which calls the move() method of Priorityscheduler to choose the next process. The move() method wakes any sleeping processes that have passed their wake-up time and pops the next process from a priority queue of processes that are ready to run. The work of switching to the new process is then performed via a native method call to the low-level virtual machine (written in C, rather than Java).

In Sect. 4, we present a model of this scheduler: a network of Circus processes interacting with the SCJ API implementation and the operating system.

\subsection{Circus}

Circus [26] is a formal notation that combines the style for data modelling of the $\mathrm{Z}[36]$ notation with that for process specification of CSP $[17,30]$. Like a CSP model, a Circus specification defines processes that communicate over channels, but that, unlike CSP processes, may contain internal state defined in Z. The internal state is encapsulated so that it can only be updated and accessed via communication on the channels of the process.

A Circus process is defined as a series of $\mathrm{Z}$ paragraphs and Circus actions, which are written using a combination of CSP constructs and Z operations. The process definition ends with a main action that defines the behaviour of the process using the actions defined previously in the process. Most CSP operators can be used in Circus actions, including external and internal choice, parallel 
Table 1. A summary of the Circus notation used in this paper

\begin{tabular}{l|l}
\hline Operator & Circus Symbol \\
\hline Prefixing of signal on channel $c$ to action $A$ & $c \longrightarrow A$ \\
\hline Prefixing of input on $c$ of value $x$ to $A$ & $c ? x \longrightarrow A$ \\
\hline Prefixing of output on $c$ of value of expression $e$ to $A$ & $c ! e \longrightarrow A$ \\
\hline Guarding of $A$ with predicate $g$ & $g \& A$ \\
\hline Termination & Skip \\
\hline External choice of actions $A$ and $B$ & $A \square B$ \\
\hline Sequential composition of $A$ and $B$ & $A ; B$ \\
\hline Interrupt of $A$ by $B$ & $A \triangle B$ \\
\hline Parallel composition $A$ and $B$ synchronising on the & $A c s 1 \|_{c s 2} B$ \\
\hline intersection of channel sets $c s 1$ and $c s 2$ & $A \| B$ \\
\hline Parallel interleaving of $A$ and $B$ & $A \backslash c s$ \\
\hline Hiding of channel set $c s$ in $A$ &
\end{tabular}

composition, sequential composition, and the interrupt operator. Additionally, Circus includes assignment, if statements, loops, and variable declarations, as well as permitting the use of $\mathrm{Z}$ schema data operations in Circus actions. Processes can also be combined using CSP operators: parallelism, hiding, and so on.

Circus has several extension, to cater for time, mobility, synchronicity and so on. Here, we use classes, included the object-oriented extension OhCircus [5].

A detailed account of Circus can be found in [26]. Examples are presented in Sect. 4. Table 1 summarises the action notation that we use here.

\section{Verification Approach}

In this section, we present our approach to verification of an SCJVM. This arises from previous experiences on modelling and verification of large existing systems [9-11]. It is, however, tailored for the needs of a VM and of an SCJVM, in particular. In this respect, this is our distillation of the lessons learned in applying Circus to reason about the icecap HVM and its scheduler. The application of the approach presented here to the scheduler is the subject of the next section.

Our technique, first of all, creates a model for a piece of code implementing a VM or a component of a VM, like a scheduler, written in any imperative or object-oriented language. Having identified the modules or classes that implement the component, our approach to modelling is in three phases: (a) data, (b) control-flow, and (c) integration modelling. These are described below.

a. Define a $Z$ Data Model.i In this phase, we formalise the data types used in the program, via the four steps below. The data types may be in the program in one of three forms: types available in the programming language, types available via a library, like the collection API of Java, or just as pieces of data not necessarily 
identified as a data type in the program. For the latter, identification of data types in the model is a matter of convenience for verification.

1. Define the state to capture the variables used in the program, creating appropriate datatypes where necessary.

2. Capture invariants that are expected. These are properties of the data model we expect the program to satisfy, even if not explicitly checked (but see b.4).

3. Define the procedures (methods, functions, and so on) of the program that manipulate the data types defined above.

4. Identify their error cases and totalise the data operations. We use theorem proving to reveal the preconditions of the operations, and extend the model to totalise those whose precondition are not just true.

By modelling errors to totalise the operations, we achieve a better understanding of the data types of the program. Its precise behaviour, which may not include checking error conditions, is captured in the next phase.

The model may be seen as a suggestion for improving the code structure (like error checking). Information from the environment of the component may also need to be identified as a type whose values are communicated in channels.

b. Capture the Control Flows Through the VM. Using the Z model, we construct a Circus model following the steps below. Roughly, each module (that is, a class, in the case of a Java program) is modelled by a Circus process or class, and its procedures by actions and methods in Circus.

1. Define channels corresponding to the services of the component. For each provided or internal service, we define a pair of channels to model calls and returns of invocations. For each required external service, we have a single channel, because we do not model its behaviour.

2. Use processes and classes to capture the modular structure of the program. A Java class should be modelled by an OhCircus class, if it includes only passive methods, that is, methods that can be modelled using only data operations without the use of channel communications, and by a process, otherwise.

3. Define the actions for the services corresponding to the channels above. In this step, we use the data operations defined in the previous phase, and capture the control flows in the definition of each action.

4. Eliminate the error cases in the $Z$ data operations that are not handled in the code, transforming the remaining cases to guards in order to enable identification of mistakes in use of data operations via deadlock checks. In this way, we ensure that the model is not more robust than the code, and any invalid assumptions about the use of the data can be revealed by analysis.

5. Define in the main action how the services are to be provided. In principle, all actions could be combined in an external choice, so that their services are available for use one at a time. A call graph, however, may identify services that are needed in parallel because they are part of different lines of execution. 
c. Generate a Circus model of the component. We use the processes defined in phase (b) to produce a model of the component as a network of processes.

1. Introduce processes to reflect the parallel design (if any) of the code.

2. Combine the various processes to define the component.

In this phase, we need to make the case that the model is closely related to the program. The argument should explain how the program modules are reflected in the structure of the $\mathrm{Z}$ data model and of processes in the network of phase (b). We need to explain how the model can be refined back to the code, and that needs to be relatively simple. For Java programs, a strong argument includes a class diagram and a mapping from the Circus processes to that diagram.

A truly faithful model defines the modelled procedures of the program as actions in Circus using programming constructs (assignments, loops, and so on) just like in the code. For reasoning, however, it is convenient to use a predicative specification of the procedures in Z. This is the reason for the abstraction in phase (a), followed by the argument constructed here. As a consequence, we catch integration problems via formal analysis, but not necessarily programming errors. The modelling effort, however, may well reveal programming errors in phases (a) and (b.4-5). In our case study, we found missing error checks.

With a model produced as described above, we open the possibility of the use of a multitude of analysis techniques. We distinguish the following possibilities as particularly useful in the case of SCJVM analysis.

1. Prove that the Circus model is deadlock free

2. Use refinement to prove more general properties.

For the icecap case study, we carry out proof of properties of the $\mathrm{Z}$ data model using Z/Eves [25], and translate the Circus model to CSP to use the FDR3 model checker [14]. In the translation, we lose the expressiveness of $\mathrm{Z}$, but gain the ability to use automatic analysis of the process network.

More details about our approach to modelling and analysis are in [12].

\section{Formal Model Overview}

We next give an overview of our model of the icecap HVM scheduler. The complete model can be found in [12, Ch. 5]; its components are shown in Fig. 3. There is a Circus process for each Java class in Fig. 2. The environment includes the low-level virtual machine written in C, the operating system, and other components of the SCJVM, including the SCJ API. These components communicate with the scheduler to initialise it and to obtain information. PrioScheduler, corresponding to the PriorityScheduler class, receives requests to move and stop SCJVM processes from PrioSchedulerImpl. It also communicates with the ClockInterruptHandler to enable and disable interrupts, and to register and start the clock handler. ClockInterruptHandler communicates with PrioSchedulerImpl to obtain the next SCJVM process to run. PrioSchedulerImpl is a bridge between ClockInterruptHandler and PrioScheduler, using services of PrioScheduler to 


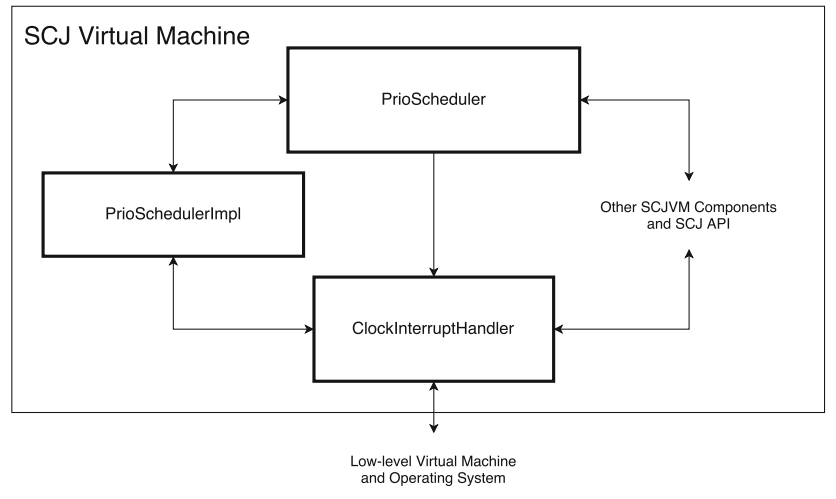

Fig. 3. SCJ VM components

determine the next process. ClockInterruptHandler also sends requests to transfer between processes to the low-level virtual machine.

Next, we describe how our model is obtained using the approach in the previous section. In phase (a), we define a $\mathrm{Z}$ data model. The most interesting types come from the class PriorityScheduler. Its state (a.1) has four parts: (i) the current time, the identifier of a ClockInterruptHandler, and a reference to an unique instance of PriorityScheduler itself ; (ii) the references to the processes managed by the scheduler; (iii) the scheduling queues containing the processes that are ready, sleeping/blocked, locked/waiting, and so on; and (iv) the SCJ event handlers managed by the scheduler. The managed processes are identified by unique elements of a set PID of identifiers used by the operating system.

The managed event handlers are modelled by the schema HandlerSet. It contains PID sets representing the different categories of handlers: periodic ( $p e h)$, aperiodic (aeh), and one shot (oseh), as well as sets of allocated (meh) and free (freeHS) handlers. It also includes a dummy identifier idle, used to avoid management of empty queues: it is queued, when the ready queue is emptied.

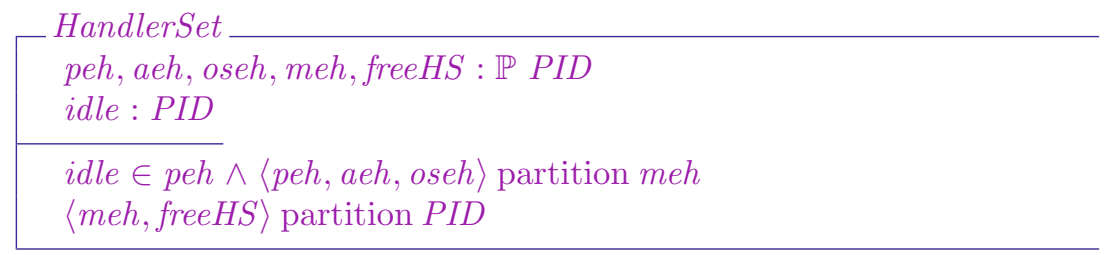

The state invariant (a.2) establishes that the $i d l e$ process is a periodic event handler, and that the allocated managed event handlers partition the different categories. Similarly, all values in PID correspond to managed processes: the allocated $(m e h)$ and free $($ free $H S)$ identifiers partition PID.

The methods corresponding to HandlerSet operations are captured as Z operations (a.3). They are simple and involve adding and removing various handlers 
from the corresponding sets, for example. Proving that the state invariant is preserved by these operations shows whether or not they are feasible. This is part of the totalisation process (a.4) that identifies error conditions to be dealt with. For example, there are operations in the scheduler that take a handler as input. There is, however, no check in the code that the input is a handler managed by the scheduler. This is an example of where we have uncovered possible issues, or hidden assumptions, of the code through modelling and proof.

In phase (b), the SCJVM-specific control-flow is captured using Circus processes. We cater for the scheduling-specific features. For illustration, we describe below the Circus basic process representing the PriorityScheduler class.

To identify the services (b.1), we perform a call-graph analysis of the nonprivate methods and create corresponding channels. As shown in Fig. 3, the scheduler interacts with the SCJ API and the operating system. A path in the call graph involving the public method transfer is as follows. Although transfer is a method of Process, because we model Process as a data type, conceptually, we regard transfer as a method of ClockInterruptHandler.

ClockInterruptHandler.run, PrioritySchedulerImpl .getNextProcess,

ClockInterruptHandler.disable, PriorityScheduler.move,

PriorityScheduler.stop, ClockInterruptHandler.transfer

The path above is part of a line of execution (and is public). So, we have a channel transfer corresponding to uses of this service. The types of the channels depend on the associated method's parameter and return types.

We follow a naming convention to identify what method call and return we are capturing. For instance, the channel KPSreleaseCall represents the package $(K)$ method of the PriorityScheduler class $(P S)$ named release that is being called. Public methods follow a similar naming, and private methods are represented with subsidiary actions, so there are no channels associated with them.

As already mentioned, for each class of the scheduler, we define a process (b.2). In defining actions (b.3), we also take advantage of the call graph. As an example, we present below the action for the release method.

$$
\begin{array}{r}
\text { Release } \widehat{=} \text { KPSreleaseCall?apeh } \longrightarrow \\
\text { PCIHdisableCall } \longrightarrow \text { PCIHdisableRet } \longrightarrow \\
(\text { pre ReleaseHandler \& ReleaseHandler }) ; \\
\text { PCIHenableCall } \longrightarrow \text { PCIHenableRet } \longrightarrow \\
\text { KPSreleaseRet } \longrightarrow \text { Skip }
\end{array}
$$

It is triggered by a call via KPSrelease Call, and concludes with a synchronisation on KPSreleaseRet. Its body contains a call to disable followed by a data operation ReleaseHandler of HandlerSet and by a call to enable. The precondition pre ReleaseHandler of ReleaseHandler is used as a guard (b.4); the input apeh is used in ReleaseHandler and its guard pre ReleaseHandler.

Another example is the action SCJStop corresponding to the method Stop. It takes an input curr, with the guard curr $\neq$ nullpid, which corresponds to the 
precondition of the method.

$$
\begin{aligned}
& \text { SCJStop } \widehat{=} \text { KPSstopCall } ? \text { curr }:(\text { curr } \neq \text { nullpid }) \longrightarrow \\
& \text { PVMtransfer!curr!mainProcess } \longrightarrow \\
& \text { KPSstopRet } \longrightarrow \text { Skip }
\end{aligned}
$$

The input curr is passed on, along with the state component mainProcess corresponding to an inherited field of the class PriorityScheduler, to the lower-level virtual machine using a channel PVMtransfer.

To conclude phase (b), we identify the services of the API that are provided in choice and in interleaving (b.5). Following the structured indicated in Fig. 3, we define that the PriorityScheduler API has three separate groups, which we name SCJApi, SCJRTE, and CIHApi, containing services provided to the SCJ infrastructure, the runtime environment and the ClockInterruptHandler. The three groups of services are combined in interleaving, with each of its constituent services in external choice. Thje choice is external, since it provides to the environment of the PriorityScheduler (see Fig. 3) the choice of which service to execute. The interleaving defines an action Run.

$$
\begin{aligned}
\text { CIHApi } & \widehat{=} \text { Move } \square \text { SCJStop } \\
\text { SCJRTE } & \widehat{=} \text { Start } \square \text { Release } \square \text { AddOuterMostSeq } \cdots \\
\text { SCJApi } & \widehat{=} \text { GetHWPrio } \square \text { GetPrio } \\
\text { Run } & \widehat{=} \text { SCJApi } \| \text { SCJRTE } \| \text { CIHApi }
\end{aligned}
$$

In the main action of PriorityScheduler, which is distinguished below by a preceding • symbol, after an initialisation using an action Init, another action Execute uses Run to provide the services of the scheduler.

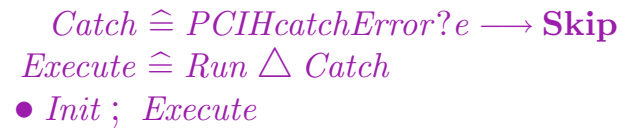

Low-level (VM) exceptions might interrupt the control flow. These exceptions may occur as a result of user-code runtime exceptions, VM-generated exceptions from environmental assumption violations (like out of memory), or residual design errors. They are indicated via a channel PCIHcatchError as defined in the action Catch, used in Execute to define the possible interruption of Run.

Finally, in phase (c) we define the Circus processes network linking together all processes representing classes from Fig. 2; it is as follows.

process Icecap VM $=\left(\begin{array}{l}\text { ClockInterruptHandler CihPsInterface } \\ \| \\ \text { PrioScheduler PSIInterface } \\ \| \text { PrioSchedulerImpl ScjPInterface } \\ \| \text { ScjProcess ScjInterface }\end{array}\right) \backslash$ csSCJRTE 
Channel sets CihPsInterface, PSIInterface, ScjPInterface, and ScjInterface are defined to include all channels used in each process. A final set containing the internal channels identified in (b.1) is used in a hiding: csSCJRTE above.

\section{Evaluation}

The icecap classes PrioScheduler and ClockInterruptHandler are modelled as processes. For other infrastructure classes, like PriorityFrame, for example, only a data model is provided, because their provided services are support operations over such data, rather than active lines of execution or SCJ provided services.

The icecap Java code associated with the component in Figs. 2 and 3 amounts to about 1600 lines of code. Following the modelling technique in Sect. 3, and illustrated in Sect.4, we obtain a Circus model presented in its entirety in [12]. There, we also find Z/Eves proofs of the totalisation of various Z schema operations, as well as a CSP version of the Circus model used for refinement and deadlock freedom checks. Table 2 provides a summary of numbers of definitions and proofs, to provide an overall total of 755 . The nature of the actual proofs using $\mathrm{Z} /$ Eves and FDR3 is discussed in the sequel.

Table 2. Summary of all Circus declarations.

\begin{tabular}{l|r|l|l}
\hline Z Declarations & Total & Circus Declarations & Total \\
\hline Unboxed items & 84 & Channel declarations & 51 \\
\hline Axiomatic definitions & 28 & Channel set declarations & 13 \\
\hline Generic axiomatic definitions & 2 & Process declarations & 12 \\
\hline Schemas & 77 & Actions & 83 \\
\hline Generic schemas & 1 & & \\
\hline Theorems & 202 & & \\
\hline Proofs & 202 & & \\
\hline
\end{tabular}

\subsection{Z/Eves Proofs}

We have used the CZT tools $[23]^{1}$ to develop the Circus model. These tools include Circus as an extension of $\mathrm{Z}$ within its Eclipse interface. CZT also integrates the Z/Eves theorem prover [31] and its proof language as an extension.

Within CZT, we have typeset (in $\mathrm{LAT}_{\mathrm{E}} \mathrm{X}$ ), typechecked and proved wellformedness conditions of the whole model. This involves theorems about functions being applied within their domain, axiomatic definitions soundness, type non-emptiness, and so on. These proofs ensure that the model is consistent.

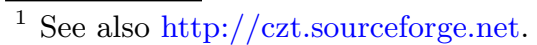


The CZT tools also have a verification-condition generator for $\mathrm{Z}$ and Circus. These include well-formedness checks (for instance, functions are called within their domains), and other consistency checks like feasibility of $\mathrm{Z}$ schema operations and race-freedom of Circus parallel actions. We have performed mechanised proofs in Z/Eves of each of these generated verification conditions.

Of greater interest are the (21) precondition proofs: they are directly related to the totalisation of operations as described by our approach (a.4). It is useful to discover the various conditions to feature in the Circus model as guards for communications. As explained, this introduces deadlocks whenever they are not satisfied. Other proofs are for well-formedness (12) and various lemmas (169) about involved types to make the precondition proofs viable.

\subsection{FDR Refinement Checks}

The Circus model is translated to CSP for automatic analysis using FDR3 [14]: a powerful refinement checker for CSP enabling automatic checking for deadlock and livelock freedom, as well as other properties of interest.

The translation strategy from Circus to CSP is beyond the scope of this paper; details about it can be found in [27]. It involves representing the $\mathrm{Z}$ data model within FDR's rich functional language, whereas the Circus CSP constructs are almost in one-to-one correspondence with those of CSP-M. Access to process state is done via channel communication and appropriate parallelism with its corresponding process main action representation in CSP.

Details about this translation for the icecap HVM scheduler model can be found in [12]. Key decisions about data abstraction and simplification of type domains are necessary to avoid state explosion. Even so, FDR can handle quite complex processes and enabled us to perform important consistency checks.

We have checked for deadlock and livelock freedom the processes related to components in Figs. 2 and 3. As expected, deadlock counterexamples occur on either events external to the components, for example, required services from the operating system, or failed precondition proofs modelled as CSP guards. The required services are for handling exceptions thrown by design or at runtime. The guards highlight hidden assumptions the icecap HVM scheduler code makes. For instance, the priority scheduler implicitly expects all processes to be known to the scheduler, and yet we can call the scheduler with "rogue" processes.

The CSP model has 540 lines excluding comments, and contains 4 top-level processes with a total of around 100 implicitly declared processes through let expressions. We use such expressions to encode Circus actions as well as state. We are still working on the process network to deal with complex state invariants, and prove more specific properties of a scheduler and of SCJ.

\section{Related Work}

There are other works on verification of real-time schedulers. Ferreira et al. [8] have worked on formal verification of the FreeRTOS scheduler using HIP/SLEEK. 
They use separation logic to verify memory safety as the FreeRTOS scheduler uses a lot of pointers, which make the use of more traditional formal verification techniques difficult. In our work, memory safety is partially guaranteed by Java's memory model and our challenges arise instead from the complex control flow of the icecap HVM scheduler. On the other hand, it has to be shown that icecap tools generate $\mathrm{C}$ code that is memory safe. This is a separate problem of compiler correctness, which is part of our agenda for future work.

A comprehensive verification of the seL4 microkernel is reported in [19]. This includes verification of the scheduler and other areas of the kernel, and a proof that the binary code of the kernel correctly implements the $\mathrm{C}$ source code. The verification of the functional properties of the system is machine-checked using a $\mathrm{C}$ semantics in Isabelle/HOL. While we focus on the icecap HVM scheduler, we expect that the icecap tools can be completely verified in the future.

For larger kernels, a major challenge in verification is the complex interdependency between the scheduler and the rest of the kernel. Gotsman and Yang [15] have developed an approach for verifying such kernels modularly using separation logic. It is also relevant to embedded systems where size and speed constraints necessitate tight coupling between operating system components. Indeed, Klein et al. [19] note that the call graph of seL4 shows high levels of interdependency between components. Gotsman and Yang demonstrate their approach by verifying a scheduler based on the Linux 2.6.11 scheduler.

We face similar challenges as the icecap tools also target embedded systems, leading to tight coupling between components, but, as said before, our challenges concern the communication between components rather than sharing of pointers. We tackle our challenges by identifying the components and specifying their interfaces. We define them as Circus processes and specify their interaction via parallel networks. Compositional reasoning and refinement can then be used.

The work of Ludwich and Fröhlich [22] verifies a system-level model of a scheduler by annotating its functions with preconditions and postconditions. These annotations constitute a formal specification that the scheduler must fulfil, which is checked using the $\mathrm{C} / \mathrm{C}++$ model checker CMBC. This work is perhaps most similar to ours due to its use of preconditions and postconditions, but our approach involves constructing a formal model from the code rather than presenting the requirements as annotations to the code.

Finally, the great value of applying formal methods in the area of scheduling is shown in [13], which reports a verification of the GCC scheduler using a model in Isabelle/HOL. This effort has uncovered a bug in the GCC Itanium scheduler that caused programs to be compiled incorrectly.

\section{Conclusion}

For SCJ to become a viable technology for use in safety-critical systems, certified runtime environments are essential. The most advanced implementation of an SCJ RTE is provided by the icecap tools. The implementation is complex. A formal model is a major step in the development of a verified RTE for SCJ. 
Developing a formal model of existing software is a major challenge. This is made more difficult with the SCJ RTE as we have to model both high and lowlevel abstractions. We have presented an approach that produces a Circus model for Java and $\mathrm{C}$ source code. Automatic construction of models is not possible, but support can be made available for encoding Java types in Z, calculation of preconditions of data operations, and extraction of call graphs, for instance.

Our experience with the icecap tools implementation has been largely positive. Although the code can be hard to fathom in places, we have found just a few bugs, mainly as the result of studying the code in sufficient depth to produce the model. There are also places where there appears to be unreachable code and where more defensive programming techniques can be employed to catch errors that can be introduced during development and maintenance.

Our experience with Circus has also been largely positive. The lack of process inheritance in Circus, however, has hindered some of our efforts. For example, for the ScjProcess class representing the abstraction for a low-level vm.Process within the SCJ paradigm, we need the gotoNextState method, which is redefined in subclasses of ScjProcess that represent periodic and aperiodic handlers and so on. Since we do not have process inheritance in Circus, in our model we have a single process ScjProcess, in which the action corresponding to gotoNextState uses a conditional to model the dynamic binding.

Our future work includes: (1) more analysis of the scheduler, for example, to show it always dispatches the highest priority SCJ event handler; (2) improvement to the code to take into account our results; (3) the analysis of other components of the icecap tools RTE, in particular the memory management module; and (4) extensions of Circus with process inheritance.

Acknowledgements. The authors gratefully acknowledge useful feedback from anonymous referees, and Stephan Erbs Korsholm and Shuai Zhao for their help in understanding the icecap HVM and its rationale. This work is supported by EPSRC Grant EP/H017461/1. No new primary data were created in this study.

Open Access. This chapter is distributed under the terms of the Creative Commons Attribution 4.0 International License (http://creativecommons.org/licenses/by/ 4.0/), which permits use, duplication, adaptation, distribution and reproduction in any medium or format, as long as you give appropriate credit to the original author(s) and the source, a link is provided to the Creative Commons license and any changes made are indicated.

The images or other third party material in this chapter are included in the work's Creative Commons license, unless indicated otherwise in the credit line; if such material is not included in the work's Creative Commons license and the respective action is not permitted by statutory regulation, users will need to obtain permission from the license holder to duplicate, adapt or reproduce the material. 


\section{References}

1. Armbruster, A., et al.: A real-time Java virtual machine with applications in avionics. ACM TECS 7(1), 5:1-5:49 (2007)

2. Atego: Atego PERC Pico - Products - Atego (2015). www.atego.com/products/ atego-perc-pico/

3. Audsley, N., Burns, A., Richardson, M., Tindell, K., Wellings, A.J.: Applying new scheduling theory to static priority pre-emptive scheduling. Softw. Eng. J. 8(5), 284-292 (1993)

4. Baxter, J.: Requirements for Safety-Critical Java Virtual Machines. Technical report, University of York (2015). http://www.cs.york.ac.uk/circus/publications/ techreports/reports/scjvm-requirements.pdf

5. Cavalcanti, A.L.C., Sampaio, A.C.A., Woodcock, J.C.P.: Unifying classes and processes. SoSyM 4(3), 277-296 (2005)

6. Cavalcanti, A.L.C., Zeyda, F., Wellings, A., Woodcock, J.C.P., Wei, K.: Safetycritical Java programs from Circus models. RTS 49(5), 614-667 (2013)

7. Dalsgaard, A.E., Hansen, R.R., Schoeberl, M.: Private memory allocation analysis for safety-critical Java. In: JTRES, pp. 9-17. ACM (2012)

8. Ferreira, J., et al.: Automated verification of the FreeRTOS scheduler in Hip/Sleek. STTT 16(4), 381-397 (2014)

9. Freitas, L., McDermott, J.P.: Formal methods for security in the Xenon hypervisor. STTT 13(5), 463-489 (2011)

10. Freitas, L., Woodcock, J.C.P.: Mechanising mondex with Z/Eves. FACJ 20(1), 117-139 (2008)

11. Freitas, L., Woodcock, J.C.P., Fu, Z.: POSIX file store in Z/Eves: an experiment in the verified software repository. SCP 74(4), 238-257 (2009)

12. Freitas, L., Cavalcanti, A., Wellings, A.: Formal specification of SCJ icecapimplementation. Technical report, Newcastle University (2015). https://www.cs. york.ac.uk/circus/publications/techreports/reports/hvm.pdf

13. Gesellensetter, L., Glesner, S., Salecker, E.: Formal verification with Isabelle/HOL in practice: finding a bug in the GCC scheduler. In: Leue, S., Merino, P. (eds.) FMICS 2007. LNCS, vol. 4916, pp. 85-100. Springer, Heidelberg (2008)

14. Gibson-Robinson, T., Armstrong, P., Boulgakov, A., Roscoe, A.W.: FDR3 - a modern refinement checker for CSP. In: Ábrahám, E., Havelund, K. (eds.) TACAS 2014 (ETAPS). LNCS, vol. 8413, pp. 187-201. Springer, Heidelberg (2014)

15. Gotsman, A., Yang, H.: Modular verification of preemptive OS kernels. JFP 23, 452-514 (2013)

16. Haddad, G., Hussain, F., Leavens, G.T.: The design of SafeJML, a specification language for SCJ with support for WCET specification. In: JTRES. ACM (2010)

17. Hoare, C.A.R.: Communicating Sequential Processes. Prentice-Hall, Upper Saddle River (1985)

18. aicas realtime.: JamaicaVM User Manual (2014). www.aicas.com/cms/en/ reference-material

19. Klein, G., et al.: Comprehensive formal verification of an OS microkernel. ACM TCS 32(1), 2:1-2:70 (2014)

20. Locke, D., Andersen, B.S., Brosgol, B., Fulton, M., Henties, T., Hunt, J.J., Nielsen, J.O., Nilsen, K., Schoeberl, M., Tokar, J., Vitek, J., Wellings, A.: Safety Critical Java Specification. The Open Group, UK (2010)

21. Luckowe, K.S., Thomsen, B., Korsholm, S.E.: HVMTP: a time predictable and portable java virtual machine for hard real-time embedded systems. In: JTRES, pp. 107-116. ACM (2014) 
22. Ludwich, M.K., Frohlich, A.A.: System-level verification of embedded operating systems components. In: SBESC, pp. 161-165, November 2012

23. Malik, P., Utting, M.: CZT: a framework for Z tools. In: Treharne, H., King, S., C. Henson, M., Schneider, S. (eds.) ZB 2005. LNCS, vol. 3455, pp. 65-84. Springer, Heidelberg (2005)

24. Marriott, C., Cavalcanti, A.: SCJ: memory-safety checking without annotations. In: Jones, C., Pihlajasaari, P., Sun, J. (eds.) FM 2014. LNCS, vol. 8442, pp. 465-480. Springer, Heidelberg (2014)

25. Meisels, I.: Software Manual for Windows Z/EVES Version 2.1. ORA Canada, tR-97-5505-04g (2000)

26. Oliveira, M.V.M., Cavalcanti, A.L.C., Woodcock, J.C.P.: A UTP semantics for Circus. FACJ 21(1-2), 3-32 (2009)

27. Oliveira, M., Sampaio, A.: Compositional analysis and design of CML models. Technical report D24.1, COMPASS, March 2013. www.compass-research.eu/ Project/Deliverables/D241.pdf

28. Pizlo, F., Ziarek, L., Vitek, J.: Real time Java on resource-constrained platforms with Fiji VM. In: JTRES, pp. 110-119. ACM (2009)

29. Richard-Foy, M., et al.: Use of PERC Pico for safety critical Java. In: ERTS (2010)

30. Roscoe, A.W.: Understanding Concurrent Systems. Texts in Computer Science. Springer, London (2011)

31. Saaltink, M.: Z/Eves 2.0 user's guide. Technical report. TR-99-5493-06a, ORA Canada (1999)

32. Søndergaard, H., Korsholm, S.E., Ravn, A.P.: Safety-critical Java for low-end embedded platforms. In: JTRES, pp. 44-53. ACM (2012)

33. Tang, D., Plsek, A., Vitek, J.: Static Checking of Safety Critical Java Annotations. In: JTRES. ACM (2010)

34. Tofte, M., Talpin, J.P.: Region-based memory management. Inf. Comput. 132(2), 109-176 (1997)

35. Wellings, A.: Concurrent and Real-Time Programming in Java. Wiley, Hoboken (2004)

36. Woodcock, J.C.P., Davies, J.: Using Z-specification, refinement, and proof. Prentice-Hall, Upper Saddle River (1996) 